\title{
A Study of the Effects on QoS in WiFi-Cellular Offloading Scenarios
}

\author{
D. Srinivasa Rao ${ }^{a}$, G. B. S. R. Naidu \\ Department of Electronics and Communication Engineering \\ G. M. R. Institute of Technology, Rajam, Srikakulam, India \\ E-mails: ${ }^{a}$ srinivasa.dasari@gmail.com, ${ }^{b}$ gbsrdecek21@gmail.com \\ ${ }^{*}$ Corresponding author
}

(Received November 5, 2018; Accepted February 24, 2019)

\begin{abstract}
Nowadays the mobile data usage has been significantly increased by an unprecedented amount with the wide spread of smart devices, which is known as the explosion of data traffic. The rapid growth in mobile data traffic leads to a deficiency of cellular network capacity. To solve this problem, readily available Wi-Fi networks are used to offload the data traffic from cellular networks. The Wi-Fi offloading must ensure guaranteed throughput and delay performance for the users. However, if the user doesn't meet any Wi-Fi network during the download period, the quality of experience gets degraded. Quality of experience can be improved with the help of various techniques such as resource allocation, scheduling, and handoff schemes. To know the effect of the offloading process, some key parameters are identified in this paper and the effect of offloading on these parameters is studied. Here, in this paper a study of various parameters like download time, number of users, data size on the throughput, delay and packet loss is done in the cellular network WiFi offloading scenarios. This study highlights the need for an efficient QoS mechanism in future heterogeneous networks. It can be considered as a research aspect in upcoming integrated networks.
\end{abstract}

Keywords- QoS, WiFi, Cellular, Offloading, Throughput, Delay, Mobile data, Access point.

\section{Introduction}

Nowadays, there is a vast increase in the number of mobile users across the globe. The internet traffic exponentially increases and the demand for video download, and other multimedia may see a huge demand in coming years. Currently, there are two major wireless networks that provide connectivity to mobile users, they are Cellular and WiFi networks. However, due to the increase in the number of mobile users, cellular networks may not provide satisfying service to the users. The only alternative is to offload the users to WiFi networks to reduce the load at the cell sites (He et al., 2016). Hence, WiFi offloading has gained a lot of attention from researchers. According to Arora et al. (2019), the offloading of mobile users to available networks is an opportunity, but we must assure the same Quality of Service (QoS) be maintained at those networks (Marchese, 2007). Generally, QoS management refers to the allocation of radio resources to requested users by providing minimum guaranteed service (Dewangan et al., 2019). QoS is usually measured in terms of some parameters such as access delay, transmission delay, throughput, jitter and packet loss rate. Although, WiFi offloading has several advantages, it should also provide the required quality to the incoming users. WiFi offloading process enables users and network operators to use the existing WiFi infrastructure without need additional investment.

These benefits can be fulfilled only by providing and maintaining the required quality of service to users. The delay introduced during the offloading process leads to a reduction of throughput among the users, and due to lack of available access points the user may lose the connection. Another key parameter which influences the QoS metric is residence time. It is the time consumed by the individual in staying network. The major objectives of this study are as follows: 
International Journal of Mathematical, Engineering and Management Sciences

Vol. 4, No. 3, 795-802, 2019

https://dx.doi.org/10.33889/IJMEMS.2019.4.3-062

- Quality metrics like throughput, delay, and packet loss were analyzed in offloading scenarios.

- The effect of various parameters on QoS has been studied in these scenarios.

- It highlights the need to develop an efficient resource allocation algorithm for future heterogeneous networks.

\section{WiFi Offloading Scenarios}

This is a recent technique used by wireless carriers that reduce usage of the cellular networks by allowing you to use your home broadband network or freely available WiFi hotspots for your mobile device data connection (Suh et al., 2016). Today's smart phones look fascinating with their networking options like mobile data using WiFi. Rather than make it harder for us to connect to WiFi network carriers are investing a lot of time and resources into making it as seamless as possible (Lui et al., 2018). To make the connectivity easy and seamless transfer, WiFi network carriers are putting a lot of efforts, time and resources. For example, operating systems like Android and iOS make it almost transparent to connect to internet using local networks automatically. Various other applications will now login to free WiFi hotspots with necessary authentication as soon as they're in range and again switching back to cellular networks when you are no longer in the coverage (Chen et al., 2016). These applications do this by automatically logging into free locations in dense places like indoor stadiums, malls, and airports (Mehmeti and Spyropoulos 2017).

Furthermore, an ever-increasing number of gadgets, for example, workstations and tablets accompany portable information broadband capacities worked in as they are intended to download content anyplace whenever. Increasing demand for bandwidth is not the only driver for WiFi offload as mobile network operators are looking for cost-effective solutions for satisfying customers (Agarwal and Tomer, 2013).

\subsection{Basic Offloading Scenario}

Besides, with an ever increasing number of new kinds of gadgets being composed particularly as WiFi emerging as the most suitable option for subscribers. The below Figure 1 depicts the WiFi offloading scenario in heterogeneous networks where WiFi Access Points (APs) are meagerly installed within cellular coverage.

The point by point strategies of every WiFi offloading method can be portrayed as takes after. Look into a scenario where a Mobile Node (MN) tries to attempt a data connection to download or upload a file from the internet at time t. It is supposed that a cellular network is constantly accessible whereas a WiFi network is only obtainable when the MN is sufficiently close to the WiFi AP (Zhou et al., 2016). On the off chance that any WiFi network is accessible at $t$ in the two methods, the MN builds up the information association just through the WiFi network in spite of the fact that a cellular network is accessible. Then again, if a WiFi network is not accessible at $\mathrm{t}$ in opportunistic WiFi offloading the mobile node uses a cellular network quickly. While for delayed WiFi offloading the portable hub concedes the information session up to a pre defined defer bound 'D'. Amid this defer bound if a WiFi network ends up accessible because of the hub versatility the MN promptly begins the pending information session through a WiFi network. Along these lines, the extra traffic can be offloaded to the WiFi network. Notwithstanding, since clients don't want to postpone the information exchange cellular network administrators give a few supportive gestures to clients to defer the information exchange to encourage deferred WiFi offloading (Dimatteo et al., 2011). 
International Journal of Mathematical, Engineering and Management Sciences

Vol. 4, No. 3, 795-802, 2019

https://dx.doi.org/10.33889/IJMEMS.2019.4.3-062

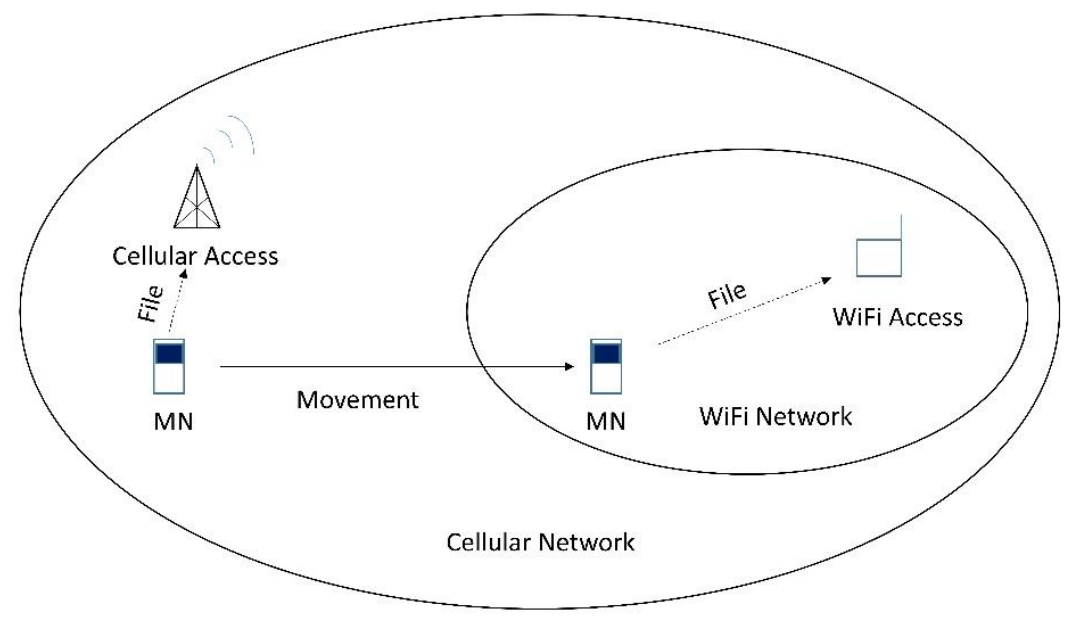

Figure 1. Basic WiFi offloading scenario

\subsection{Offloading Scenarios}

The offloading process from Cellular network to WiFi can be made depending on the user residence time. In this paper, we studied two scenarios. One is an evaluation of QoS metrics when the user is under the residence of both cellular and WiFi networks, another is when the user is under the presence of only WiFi terminals. We studied the effect of some key parameters on the QoS in these two cases. The illustration of the two cases is shown in Figure 2.

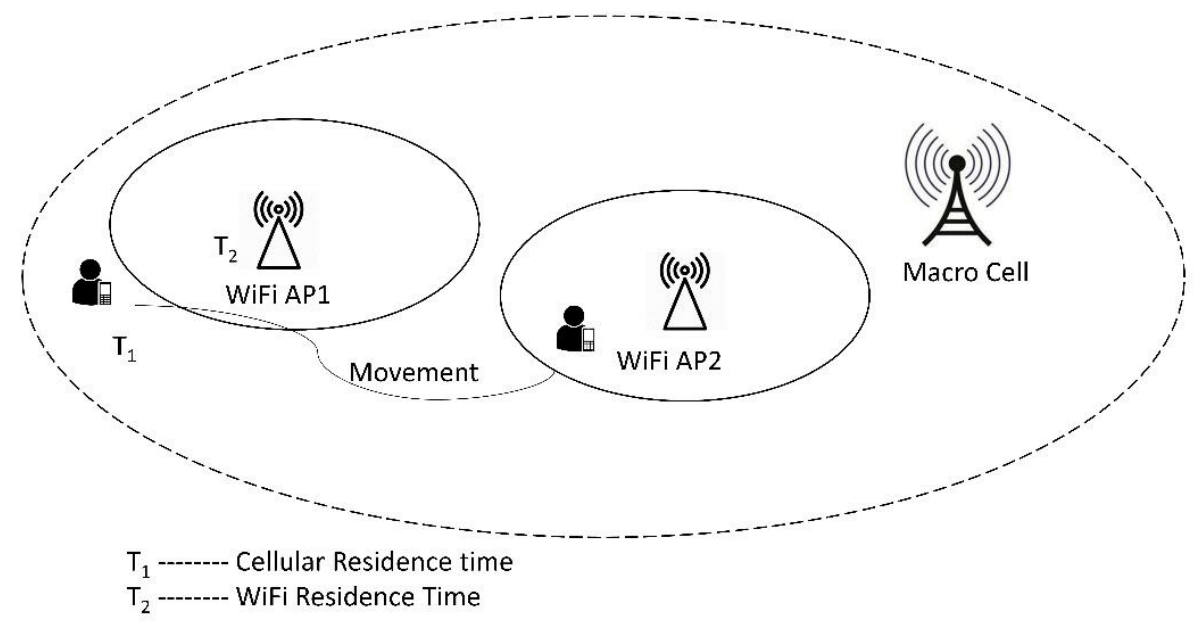

Figure 2. User movement over heterogeneous networks

Initially, the user is under the coverage of WiFi AP 1 . However, if the user stays a short time in AP 1, then while moving from AP1 to AP2, the downloading process continues under the macro cell. At time instant T1, the user could able to get service due to dual connectivity. The user could lose 
International Journal of Mathematical, Engineering and Management Sciences

Vol. 4, No. 3, 795-802, 2019

https://dx.doi.org/10.33889/IJMEMS.2019.4.3-062

connectivity when he is far away from another AP or if the delay is more compared to the distance of separation. The file transfer should take place to maintain the QoS promised in cellular networks. That means the File transfer protocol (FTP) has deadlines required to be satisfied. The offloading scenarios considered above depicts that, when the users move away from the APs the connectivity would be lost and regained with dual connectivity approaches. However, delayed offloading leads to degradation of the performance of the entire system. Due to this, a noteworthy test is to evaluate the extra limit brought by the utilization of offloading techniques. The most remarkable impact of offloading ought to be the decrease of traffic load and blockage in the essential system. Also, limit enhancements depend, in addition to other things, on the quantity of cell phones or remote APs engaged with the procedure, on the portability of hubs, on the size and the delay acceptance of the offloaded content. Then again, client fulfillment is frequently related to Quality of Service, so the acquired throughput and obtained metrics are viewed as the most vital parameters. Hence, there is a need to develop efficient QoS enhancement technique during the offloading process.

\section{Study of Key Parameters}

To study the performance of these networks, we considered some key parameters like a number of users, payload size, and download time. This section describes these parameters in details.

\subsection{Impact of Number of Users}

Due to an increase in the demand for data traffic, the number of users downloading the data also increases. In places like indoor stadiums, malls, airport the number of users consuming the internet is increasing. Hence, the resources should be allocated efficiently and the overall throughput must be maximized. Here, in this, the effect of a number of users on throughput is studied.

\subsection{Impact of Data Size}

Multimedia traffic involves the transfer of huge amounts of data. For, delay tolerant applications this doesn't show great effect. However, for applications like video calls and conference calls, the data size has a significant effect on the quality. Hence, this is a major parameter that must be taken care of while offloading the users. The performance of these networks with respect to data size is studied.

\subsection{Impact of Download Time}

The downloading time is also a major factor in current heterogeneous networks. As, the user moves between various networks, the session will be disconnected and initiated at different time instants. Due to this download of a small amount of data may also require large time. This introduces a delay in various applications. Here, we considered the relation between data size and download time.

The above parameters represent the major metrics to measure the performance of any cellular network. (Hu et al., 2016). There is a need to optimize the throughput of the system with the increase in the number of users and their QoS demands.

\section{Results and Discussion}

The analysis of various parameters is carried out using an event-driven simulator based on MATLAB and conducted simulations (Daryl, 2014). The following results show the effect of various parameters on QoS metrics. This section gives a discussion of these effects. Table 1 shows the simulation parameters used. 
International Journal of Mathematical, Engineering and Management Sciences

Vol. 4, No. 3, 795-802, 2019

https://dx.doi.org/10.33889/IJMEMS.2019.4.3-062

Table 1. Simulation parameters

\begin{tabular}{|c|c|c|}
\hline S. No & Parameter & Value \\
\hline 1 & Coverage area & $100 \mathrm{~m}$ \\
\hline 2 & Number of stations & $10-50$ \\
\hline 3 & Station movement & Random \\
\hline 4 & Channel Bandwidth & Spatial spreading \\
\hline 5 & Transmission scheme & $2.4 \mathrm{GHz}$ \\
\hline 6 & Operating frequency & \\
\hline
\end{tabular}

Figure 3 shows that the throughput decreases with an increase in the number of users in higher density scenarios. This shows that the user throughput will be degraded during offloading and highdensity scenarios. This implies that the throughput performance degrades when the user moves from one AP to another AP, as this involves large delay. The user would lose connectivity when the distance of separation is large between the APs. Hence, dual connectivity shows better performance with the increase in the number of users.

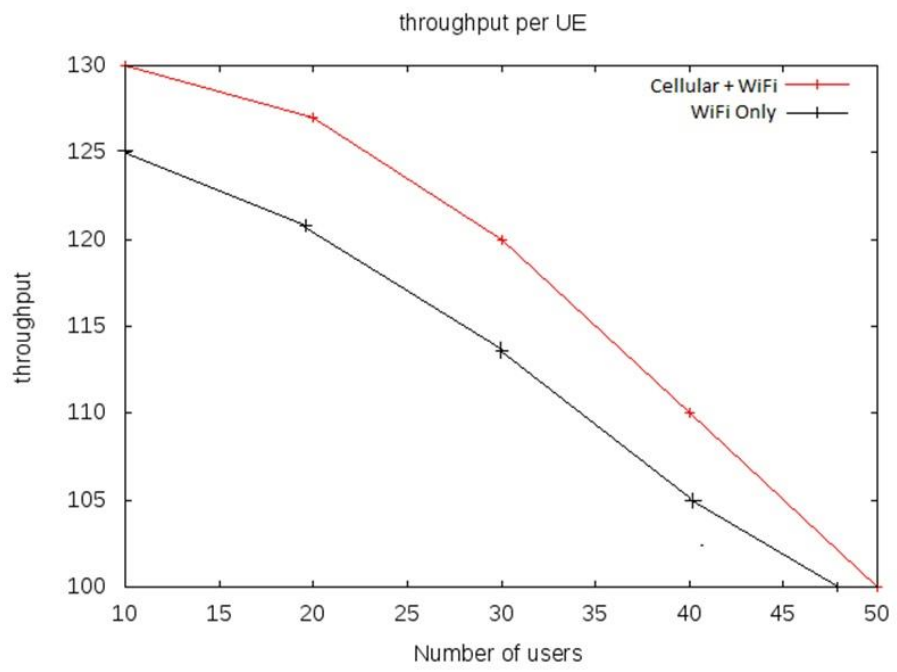

Figure 3. Throughput vs. number of users

Figure 4 shows the effect of timing on delay. Packet delay will be high when the channel conditions are bad and if the connectivity doesn't take place even after threshold time. As seen from the throughput performance, it can be shown that the delay will be more when the distance of separation is large between two Aps. 
International Journal of Mathematical, Engineering and Management Sciences

Vol. 4, No. 3, 795-802, 2019

https://dx.doi.org/10.33889/IJMEMS.2019.4.3-062

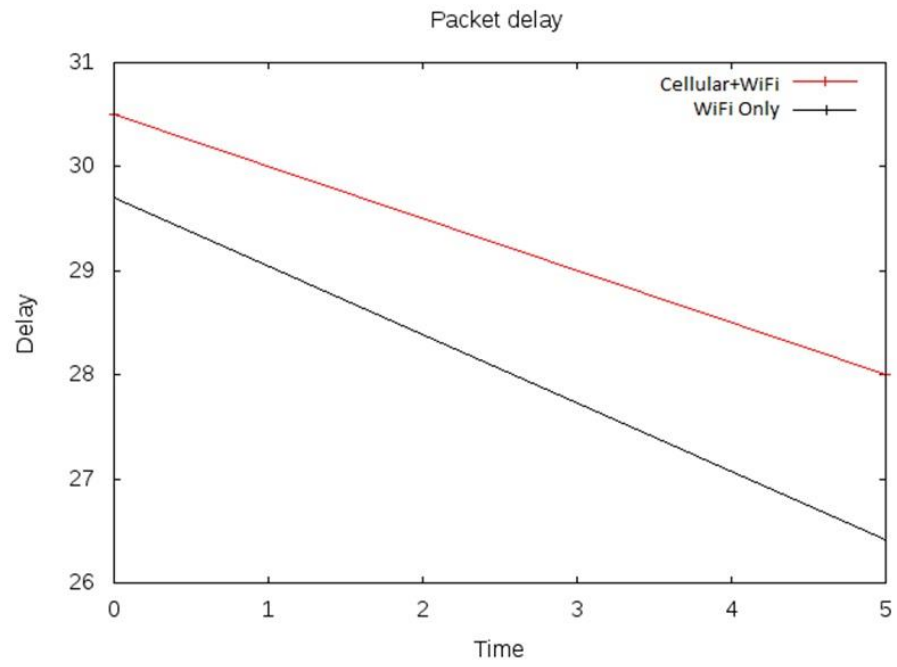

Figure 4. Time vs. delay

Figure 5 shows the result of packet loss. The result shows that the packet loss is more when the connection of the data is not initiated in a prescribed time. Hence, during the offloading process, the QoS should be well maintained to preserve the quality to users. Due to delay in the connectivity, there could be a loss of packets that request from the users. Hence, to reduce the loss of packets, the connection should be established within less time.

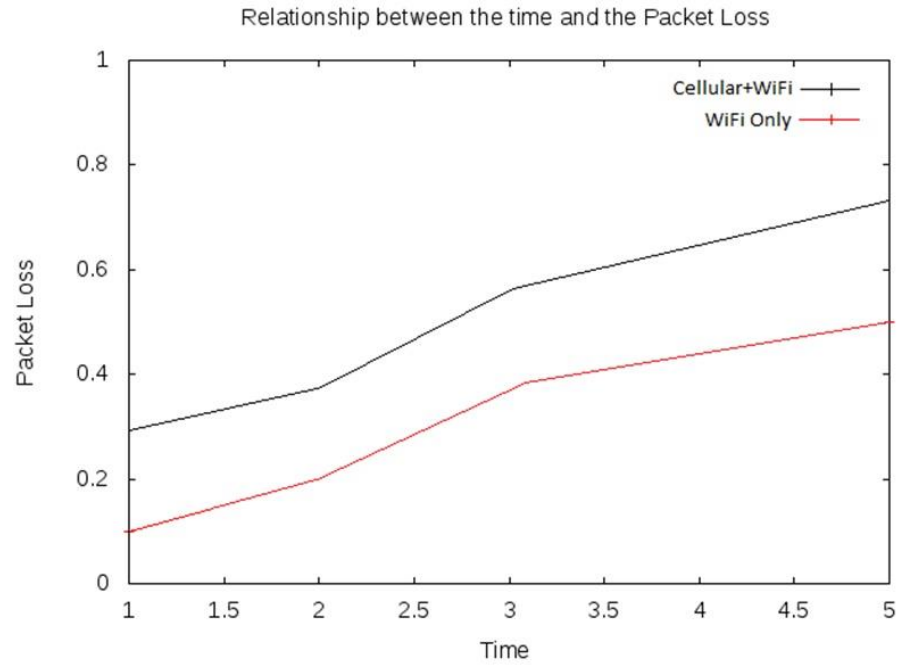

Figure 5. Time vs. packet loss

Figure 6 shows that the download time increases with the increase in data packet size. This is because the download time is mainly affected by data size rather the delay in accessing the network. Likewise, it can be observed that the difference between the available networks and with only WiFi 
International Journal of Mathematical, Engineering and Management Sciences

Vol. 4, No. 3, 795-802, 2019

https://dx.doi.org/10.33889/IJMEMS.2019.4.3-062

connection is represented in the graph. This is on account of there will be more possibilities that the client downloads information with the double network when data packet size is huge. That is the impact of double availability blows up evident as packet size increases. The trade-off among the two schemes discussed is given in Table 2 with respect to various QoS metrics.

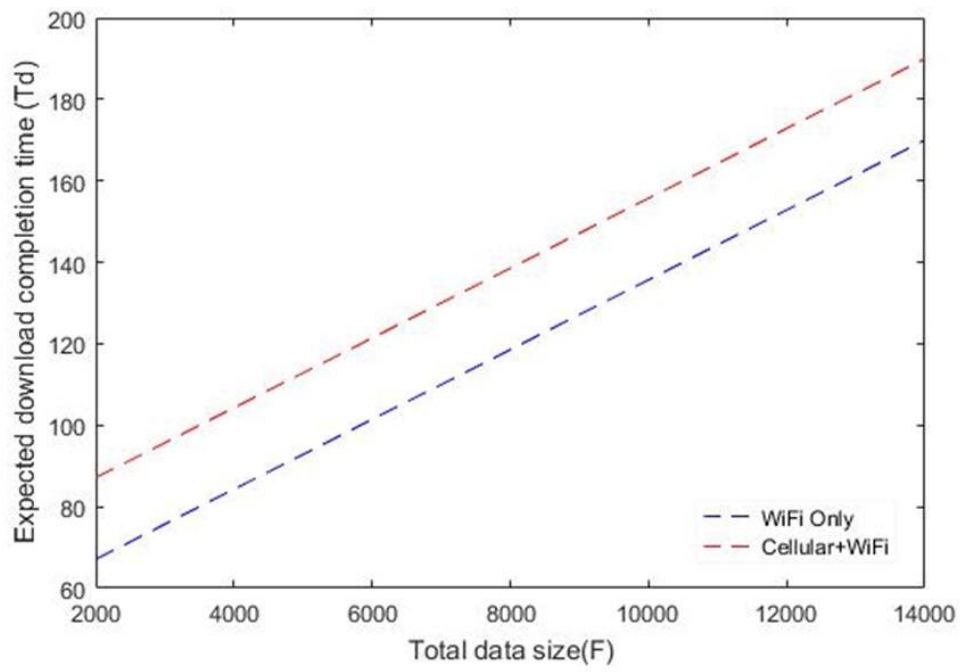

Figure 6. Data size vs. download time

Table 2. Simulation parameters

\begin{tabular}{|c|c|c|c|}
\hline S. No & Metrics & Cellular+WiFi & WiFi Only \\
\hline 1 & Throughput & High & Low \\
\hline 2 & Delay & High & Low \\
\hline 3 & Packet Loss & High \\
\hline
\end{tabular}

\section{Conclusion}

Offloading is a technique to reduce the load on some networks, and offers significant improvement of coverage and capacity. Although, this technique offers great advantages, it also places questions like, re-initiation time, sustaining QoS metrics that were promised in cellular networks. Hence, in order to realize the benefits of WiFi offloading it is necessary to eliminate these problems. Here, in this paper, we studied the effect of number of users, download time and payload size on the performance of these types of networks. In summary, the QoS offered by the cellular networks degrades during the offloading process, it shows that there is the need to develop an efficient resource management technique in heterogeneous networks. This helps readers to develop an interest in developing algorithms and techniques that speed up the process of offloading among networks. 
International Journal of Mathematical, Engineering and Management Sciences

Vol. 4, No. 3, 795-802, 2019

https://dx.doi.org/10.33889/IJMEMS.2019.4.3-062

\section{Conflict of Interest}

The authors confirm that this article contents has no conflict of interest.

\section{Acknowledgment}

The authors acknowledge and express the gratitude for the support of GMR Institute of Technology, Rajam, India.

\section{References}

Agarwal, R., \& Tomer, A. (2013). Carrier Wi-Fi offload: charting the road ahead. Tata Consultancy Services White Paper, 1-14.

Arora, A., Rakesh, N. \& Mishra, K.K. (2019). Asset distribution and peerless selection approach for LTE based multifarious VANET networks. International Journal of Mathematical, Engineering and Management Sciences, 4(1), 27-40.

Dewangan, B.K., Agarwal, A., Venkatadri, M., \& Pasricha, A. (2019). Energy-Aware Autonomic Resource Scheduling Framework for Cloud. International Journal of Mathematical, Engineering and Management Sciences, 4(1), 41-55.

Liu, B., Zhu, Q., \& Zhu, H. (2018). Delay-aware LTE WLAN aggregation in heterogeneous wireless network. IEEE Access, 6, 14544-14559.

Daryl, N. (2014). Modelling and analysis of discrete event simulations. The MathWorks, Inc.

Suh, D., Ko, H., \& Pack, S. (2016). Efficiency analysis of WiFi offloading techniques. IEEE Transactions on Vehicular Technology, 65(5), 3813-3817.

Mehmeti, F., \& Spyropoulos, T. (2017). Performance analysis of mobile data offloading in heterogeneous networks. IEEE Transactions on mobile computing, 16(2), 482-497.

Xu, H., Wen, X., Lu, Z., Hu, Z., Jing, W., \& Chen, K. (2016, September). Performance analysis of delayed mobile data offloading with multi-level priority. In 2016 IEEE 27th Annual International Symposium on Personal, Indoor, and Mobile Radio Communications (PIMRC) (pp. 1-6). IEEE.

Marchese, M. (2007). QoS over heterogeneous networks. John Wiley \& Sons.

Chen, Q., Yu, G., Shan, H., Maaref, A., Li, G. Y., \& Huang, A. (2016). Cellular meets WiFi: Traffic offloading or resource sharing?. IEEE Transactions on Wireless Communications, 15(5), 3354-3367.

Dimatteo, S., Hui, P., Han, B., \& Li, V. O. (2011). Cellular traffic offloading through WiFi networks. In IEEE International Conference on Mobile Adhoc and Sensor Systems Proceedings (pp. 192-201). IEEE Computer Society.

He, Y., Chen, M., Ge, B., \& Guizani, M. (2016). On WiFi offloading in heterogeneous networks: Various incentives and trade-off strategies. IEEE Communications Surveys \& Tutorials, 18(4), 2345-2385.

Zhou, Z., Teng, F., Liu, J., \& Xiao, W. (2016, February). Performance evaluation for coexistence of LTE and WiFi. In 2016 International Conference on Computing, Networking and Communications (ICNC) (pp. 1-6). IEEE. 